\title{
The Influence of Hepatic and Renal Impairment on the Pharmacokinetics of a Treatment for Herpes Zoster, Amenamevir (ASP2151): Phase 1, Open-Label, Single-Dose, Parallel-Group Studies
}

\author{
Tomohiro Kusawake · Donna Kowalski · Akitsugu Takada • \\ Kota Kato - Masataka Katashima · James J. Keirns • Michaelene Lewand • \\ Kenneth C. Lasseter · Thomas C. Marbury · Richard A. Preston
}

Received: September 25, 2017 / Published online: November 13, 2017

(C) The Author(s) 2017. This article is an open access publication

\begin{abstract}
Introduction: Amenamevir (ASP2151) is a nonnucleoside human herpesvirus helicase-primase inhibitor that was approved in Japan for the treatment of herpes zoster (shingles) in 2017. This article reports the results of two clinical trials that investigated the effects of renal and hepatic impairment on the pharmacokinetics of amenamevir.

Methods: These studies were phase 1, open-label, single-dose (oral $400 \mathrm{mg}$ ), parallel-group studies evaluating the pharmacokinetics, safety, and tolerability of amenamevir in healthy participants and participants with moderate
\end{abstract}

Enhanced content To view enhanced content for this article go to http://www.medengine.com/Redeem/ 10DCF0602A433BEC.

T. Kusawake $(\varangle) \cdot$ D. Kowalski · J. J. Keirns .

M. Lewand

Astellas Pharma Global Development Inc.,

Northbrook, IL, USA

e-mail: tomohiro.kusawake@astellas.com

A. Takada $\cdot$ M. Katashima

Astellas Pharma Inc., Tokyo, Japan

K. Kato

Astellas Pharma Inc., Tsukuba, Japan

K. C. Lasseter

Clinical Pharmacology of Miami LLC, Miami, FL, USA hepatic impairment and mild, moderate, and severe renal impairment.

Results: In the hepatic impairment study, the pharmacokinetic profile of amenamevir in participants with moderate hepatic impairment was generally similar to that of participants with normal hepatic function. In the renal impairment study, the area under the amenamevir concentration versus time curve from the time of dosing up to the time of the last sample with extrapolation to infinity of the terminal phase was increased by $78.1 \%$ in participants with severe renal impairment. There was a positive relationship between creatinine clearance and oral and renal clearance for amenamevir in the renal impairment study. In both studies, amenamevir was safe and well tolerated.

T. C. Marbury

Orlando Clinical Research Center, Orlando, FL, USA

R. A. Preston

Division of Clinical Pharmacology, University of Miami, Miami, FL, USA

R. A. Preston

Department of Cellular Biology and Pharmacology, Florida International University, Miami, FL, USA 
Conclusion: The findings of the hepatic impairment study indicate that no dosing adjustment is required in patients with moderate hepatic impairment. In the renal impairment study, systemic amenamevir exposure was increased by renal impairment. However, it is unlikely that renal impairment will have a significant effect on the safety of amenamevir given that in previous pharmacokinetic and safety studies in healthy individuals amenamevir was safe and well tolerated after a single dose (5-2400 mg, fasted condition) and repeated doses for 7 days (300 or $600 \mathrm{mg}$, fed condition), and the amount of amenamevir exposure in the renal impairment study was covered by those studies. These findings suggest that amenamevir does not require dosage reduction in accordance with the creatinine clearance

Funding: Astellas Pharma.

Keywords: Amenamevir; Hepatic impairment; Herpes zoster; Pharmacokinetics; Renal impairment; Safety

\section{INTRODUCTION}

Herpes zoster, also called shingles, is a painful rash that is caused by reactivation of the varicella-zoster virus (VZV) in cranial nerve or dorsal root ganglia, with spread of the virus along the sensory nerve to the dermatome [1]. More than $95 \%$ of immunocompetent individuals aged 50 years or older are seropositive for VZV and are at risk of developing herpes zoster [2]. The incidence increases markedly with age, and the lifetime risk of developing herpes zoster is estimated to be $10-20 \%$ in the general population and as high as $50 \%$ of people who live to 85 years [3].

Nucleoside analogues such as acyclovir [4], valacyclovir [5], and famciclovir [6] have been approved for the treatment of herpes simplex virus 1 , herpes simplex virus 2 , and VZV infections. Acyclovir, valacyclovir, and famciclovir are mainly excreted by the kidney, with a study indicating that the famciclovir area under the concentration versus time curve from the time of dosing up to the time of the last sample with extrapolation to infinity of the terminal phase $\left(\mathrm{AUC}_{\mathrm{inf}}\right)$ was increased by about $6.8 \%, 218.0 \%$, and $766.2 \%$ in individuals with mildly, moderately, and severely impaired renal function, respectively, compared with individuals with normal renal function [7]. Because of reduced clearance and the resulting increased exposure in patients with renal dysfunction, dosage reduction is recommended for patients with creatinine clearance less than $25 \mathrm{~mL} / \mathrm{min} / 1.73 \mathrm{~m}^{2}$, 50 , and $60 \mathrm{~mL} / \mathrm{min}$ for acyclovir, valacyclovir, and famciclovir. Renal function decreases with advancing age, and significant renal dysfunction is more common in people aged 60 years or older [8]. People of this age group are also more likely to develop herpes zoster [3] and therefore constitute the major target population for antiviral therapy. Accordingly, a drug with a more simplified dosing schedule requiring less consideration of renal function is desirable.

Amenamevir (ASP2151) is an oxadiazolephenyl-derived nonnucleoside human herpesvirus helicase-primase inhibitor with more potent in vitro antiviral activity against VZV than acyclovir [9]. The helicase-primase complex has an essential function for viral genomic replication, responsible for both unwinding viral DNA at the replication fork and priming DNA synthesis [10-14]. The main elimination pathway of amenamevir was hepatic metabolism in animal studies (unpublished data). In a study in healthy volunteers, urinary excretion as unchanged drug was approximately 10\% [15].

This article reports the results of two clinical trials that investigated the effects of renal and hepatic impairment on the pharmacokinetics of amenamevir.

\section{METHODS}

\section{Study Design}

These studies were phase 1, open-label, single-dose, parallel-group studies evaluating the pharmacokinetics, safety, and tolerability of amenamevir in healthy participants and participants with moderate hepatic impairment or various degrees of renal impairment. These 
studies were not registered at a registry website as there was no requirement in the International Council for Harmonisation of Technical Requirements for Pharmaceuticals for Human Use (ICH) "Guideline for Good Clinical Practice" to register phase 1 trials at the time these studies were conducted.

The hepatic impairment study (study 15L-CL-013) was conducted at Orlando Clinical Research Center (Orlando, FL, USA), and the renal impairment study (study 15L-CL-014) was performed at four sites in the USA: Orlando Clinical Research Center, the University of Miami (Miami, FL), DGD Research (San Antonio, TX), and Clinical Pharmacology of Miami (Miami, FL).

Before initiation of the studies, the clinical study protocols were approved by the Independent Investigational Review Board (Plantation, FL, USA) for Orlando Clinical Research Center and Clinical Pharmacology of Miami, the University of Miami Human Subjects Research Office (Miami, FL, USA) for the University of Miami, and RCRC IRB (Austin, TX, USA) for DGD Research. The studies were conducted in accordance with the ethical standards of the ICH "Guideline for Good Clinical Practice." All procedures followed were in accordance with the ethical standards of the responsible committee on human experimentation (institutional and national) and with the Helsinki Declaration of 1964, as revised in 2000. Written informed consent was obtained from all participants for their being included in these studies.

\section{Study Participants}

For the hepatic impairment study, male and female participants, 18-75 years of age, with a body mass index (BMI) between 18 and $40 \mathrm{~kg} /$ $\mathrm{m}^{2}$ were eligible for enrollment if they were in good health (healthy participants) or had stable moderate hepatic impairment (defined as a predose hepatic assessment of class B as defined by the Child-Pugh classification [16]). For the renal impairment study, male and female participants, 18-79 years of age, with a BMI between 18 and $40 \mathrm{~kg} / \mathrm{m}^{2}$ were eligible for enrollment if they were in good health (healthy participants) or had stable renal impairment. Participants were categorized on the basis of their predose Cockcroft-Gault estimated creatinine clearance: more than $80 \mathrm{~mL} / \mathrm{min}$ was considered normal renal function, $50-80 \mathrm{~mL} /$ min was considered mild renal impairment, 30 to less than $50 \mathrm{~mL} / \mathrm{min}$ was considered moderate renal impairment, and less than $30 \mathrm{~mL} / \mathrm{min}$ was considered severe renal impairment. This study started in November 2007 and followed the US Food and Drug Administration's "Guidance for Industry" [17] for the study's renal impairment criteria.

\section{Dosing and Sampling Schedules}

For both studies, participants received a single oral 400-mg dose of amenamevir as two tablets (200 mg each) with $240 \mathrm{~mL}$ of water in the morning after a minimal 8-h fast. The 400-mg dose of amenamevir was selected for these studies on the basis of the pharmacokinetic estimation of the clinical dose.

Blood samples were collected for measurement of amenamevir concentrations at $15 \mathrm{~min}$ before dosing and $0.25,0.5,1,1.5,2,3,4,6,8$, $10,12,16,24,36,48,72$, and 96 h after study drug administration. Blood samples were also collected at 2 and $8 \mathrm{~h}$ after study drug administration for analysis of amenamevir binding to plasma protein. Urine samples were collected for measurement of amenamevir concentrations within $30 \mathrm{~min}$ (hepatic impairment study) or $12 \mathrm{~h}$ (renal impairment study) before dosing, and $0-4 \mathrm{~h}, 4-8 \mathrm{~h}, 8-12 \mathrm{~h}, 12-24 \mathrm{~h}, 24-36 \mathrm{~h}$, 36-48 h, 48-72 h, and 72-96 h after dosing.

\section{Pharmacokinetic Assessments}

Unchanged amenamevir and monohydroxy metabolite R5 concentrations in plasma were measured by a validated liquid chromatography-tandem mass spectrometry method at Covance Laboratories. Amenamevir and R5 were isolated from plasma by liquid-liquid extraction using tert-butyl methyl ether. Deuterium-labeled amenamevir and R5 were used as 
the internal standards. The extracts were analyzed by liquid chromatography-tandem mass spectrometry, using isocratic elution, with $40 \%$ $0.1 \%$ formic acid in water and $60 \% 0.1 \%$ formic acid in methanol, on a Chromolith SpeedROD RP-18e $50 \mathrm{~mm} \times 4.6 \mathrm{~mm}$ column, with use of a Sciex API 4000 system with a turbo ion spray interface in positive ion mode. The method was validated over a range of $5-5000 \mathrm{ng} / \mathrm{mL}$ for amenamevir and 2-2000 $\mathrm{ng} / \mathrm{mL}$ for R5 with use of $0.1 \mathrm{~mL}$ plasma.

The primary pharmacokinetic parameters assessed in both studies were $\mathrm{AUC}_{\text {inf }}$ and the maximum observed concentration $\left(C_{\max }\right)$. Secondary pharmacokinetic parameters assessed in both studies included half-life $\left(t_{1 / 2}\right)$, the time to maximum observed concentration $\left(t_{\max }\right)$, oral clearance, fraction of unbound drug, renal clearance, and the amount of drug excreted into urine up to the time of collection of the last measurable concentration $\left(\mathrm{Ae}_{\text {last }}\right)$.

Parameters were calculated by noncompartmental analysis with Phoenix ${ }^{\circledR}$ WinNonlin ${ }^{\circledR}$ version 5.3 (Certara USA, Princeton, NJ, USA).

\section{Safety Assessments}

For both studies, safety assessments included the evaluation of the frequency and severity of treatment-emergent adverse events (TEAEs), 12-lead electrocardiogram (ECG) recordings, clinical laboratory tests (biochemistry, hematology, serology, and urinalysis), vital signs (blood pressure and pulse), and physical examination findings. TEAEs were coded according to the Medical Dictionary for Regulatory Activities (MedDRA) version 9.1, and were summarized by system organ class and preferred term.

\section{Statistical Analysis}

A planned sample size of 16 participants (eight per group) for the hepatic impairment study and 32 participants (eight per group) for the renal impairment study was determined on the basis of precedent set by other pharmacokinetic studies similar in design.
Descriptive statistics (number of participants, mean, and standard deviation) were used to summarize continuous variables, and frequency and percentage were used to describe categorical variables. For each study, to assess the effect of renal or hepatic impairment on the pharmacokinetics of amenamevir, an analysis of variance was performed on natural-log-transformed $\mathrm{AUC}_{\mathrm{inf}}$ and $C_{\max }$. Geometric least squares means were used to calculate the ratios of pharmacokinetic parameters in the impaired groups to those in the normal control groups, along with 90\% confidence intervals (CIs). Similar analyses were conducted for metabolite R5. Linear regression was used to evaluate the relationships between selected pharmacokinetic parameters (oral clearance and renal clearance) and estimated renal function (creatinine clearance). Safety data were analyzed with use of descriptive statistics. All data processing, summarization, and analyses were performed with SAS $^{\circledR}$ version 9.1 (SAS, Cary, NC, USA).

\section{RESULTS}

\section{Study Population}

In the hepatic impairment study, 16 participants (eight participants with normal hepatic function and eight participants with moderately impaired hepatic function) were enrolled. In the renal impairment study, 33 participants (nine participants with normal renal function, eight participants with mild renal impairment, eight participants with moderate renal impairment, and eight participants with severe renal impairment) were enrolled. For both studies, all participants (16 participants for the hepatic impairment study and 33 participants for the renal impairment study) completed the study and were evaluable for assessment of safety and pharmacokinetics.

The demographics and baseline characteristics of the participants in the two studies are summarized in Table 1 . The participants with normal hepatic and renal function were matched with participants with hepatic and renal impairment by approximate age, sex, and BMI of the participants. 
Table 1 Baseline characteristics

\begin{tabular}{|c|c|c|c|c|c|c|}
\hline \multirow[t]{2}{*}{ Study } & \multicolumn{2}{|c|}{ Hepatic impairment study } & \multicolumn{4}{|c|}{ Renal impairment study } \\
\hline & $\begin{array}{l}\text { Normal hepatic } \\
\text { function } \\
(n=8)\end{array}$ & $\begin{array}{l}\text { Moderate hepatic } \\
\text { impairment } \\
(n=8)\end{array}$ & $\begin{array}{l}\text { Normal renal } \\
\text { function } \\
(n=9)\end{array}$ & $\begin{array}{l}\text { Mild renal } \\
\text { impairment } \\
(n=8)\end{array}$ & $\begin{array}{l}\text { Moderate renal } \\
\text { impairment } \\
(n=8)\end{array}$ & $\begin{array}{l}\text { Severe renal } \\
\text { impairment } \\
(n=8)\end{array}$ \\
\hline \multicolumn{7}{|l|}{ Sex } \\
\hline Male & $6(75 \%)$ & $6(75 \%)$ & $4(44.4 \%)$ & $3(37.5 \%)$ & $4(50 \%)$ & $5(62.5 \%)$ \\
\hline Female & $2(25 \%)$ & $2(25 \%)$ & $5(55.6 \%)$ & $5(62.5 \%)$ & $4(50 \%)$ & $3(37.5 \%)$ \\
\hline $\begin{array}{l}\text { Age } \\
\text { (years) }\end{array}$ & $52.3 \pm 7.8$ & $53.3 \pm 5.4$ & $55.2 \pm 16.3$ & $62.0 \pm 15.0$ & $68.4 \pm 16.5$ & $67.3 \pm 6.7$ \\
\hline $\begin{array}{c}\text { Weight } \\
(\mathrm{kg})\end{array}$ & $76.6 \pm 15.4$ & $81.9 \pm 17.4$ & $76.2 \pm 9.5$ & $73.7 \pm 21.0$ & $75.0 \pm 14.5$ & $80.4 \pm 20.1$ \\
\hline $\begin{array}{l}\text { Height } \\
\text { (m) }\end{array}$ & $1.75 \pm 0.07$ & $1.75 \pm 0.08$ & $1.67 \pm 0.10$ & $1.67 \pm 0.12$ & $1.63 \pm 0.05$ & $1.63 \pm 0.09$ \\
\hline $\begin{array}{l}\text { BMI } \\
(\mathrm{kg} / \\
\left.\mathrm{m}^{2}\right)\end{array}$ & $25.1 \pm 4.5$ & $26.8 \pm 5.8$ & $27.3 \pm 3.9$ & $26.3 \pm 6.0$ & $27.9 \pm 4.1$ & $29.7 \pm 4.7$ \\
\hline
\end{tabular}

All values are presented as the mean \pm standard deviation unless otherwise stated $B M I$ body mass index

\section{Pharmacokinetics}

\section{Effect of Hepatic Impairment}

Mean plasma concentration-time profiles for amenamevir and metabolite R5 in participants with normal and moderately impaired hepatic function after oral administration of a 400-mg dose of amenamevir are presented in Fig. 1. Summary statistics for amenamevir and metabolite R5 pharmacokinetic parameters in plasma and urine for participants with normal hepatic function and participants with moderate hepatic impairment are presented in Table 2. In participants with moderate hepatic impairment, there was no essential change in mean $\mathrm{AUC}_{\text {inf }}$ and $C_{\text {max }}$ of amenamevir $(4.3 \%$ and $9 \%$ decrease, respectively) compared with participants with normal hepatic function. Somewhat larger decreases of $25.2 \%$ and $32.5 \%$, respectively, were seen for metabolite R5 (Table 2). The mean elimination $t_{1 / 2}$ of amenamevir in participants with normal and moderately impaired hepatic function was 7.7 and $8.9 \mathrm{~h}$, respectively. The mean oral clearance of amenamevir in participants with normal hepatic function $(28.6 \mathrm{~L} / \mathrm{h})$ and in participants with moderately impaired hepatic function (28.4 $\mathrm{L} / \mathrm{h}$ ) was similar. There was no difference in the mean fraction of unbound amenamevir between participants with normal hepatic function and participants with impaired hepatic function (22.1\% and 23.0\%, respectively).

The percentage $\mathrm{Ae}_{\text {last }}$ was not different between participants with normal hepatic function $(9.6 \%)$ and those with moderately impaired hepatic function (9.8\%). For R5, Ae last was $25 \%$ lower in participants with moderately impaired hepatic function compared with participants with normal hepatic function.

\section{Effects of Renal Impairment}

Mean plasma concentration-time profiles for amenamevir and metabolite R5 in participants with mild, moderate, and severe renal impairment and normal renal function are shown in Fig. 2, and summary statistics for amenamevir and metabolite R5 pharmacokinetic parameters in plasma and urine for participants with 

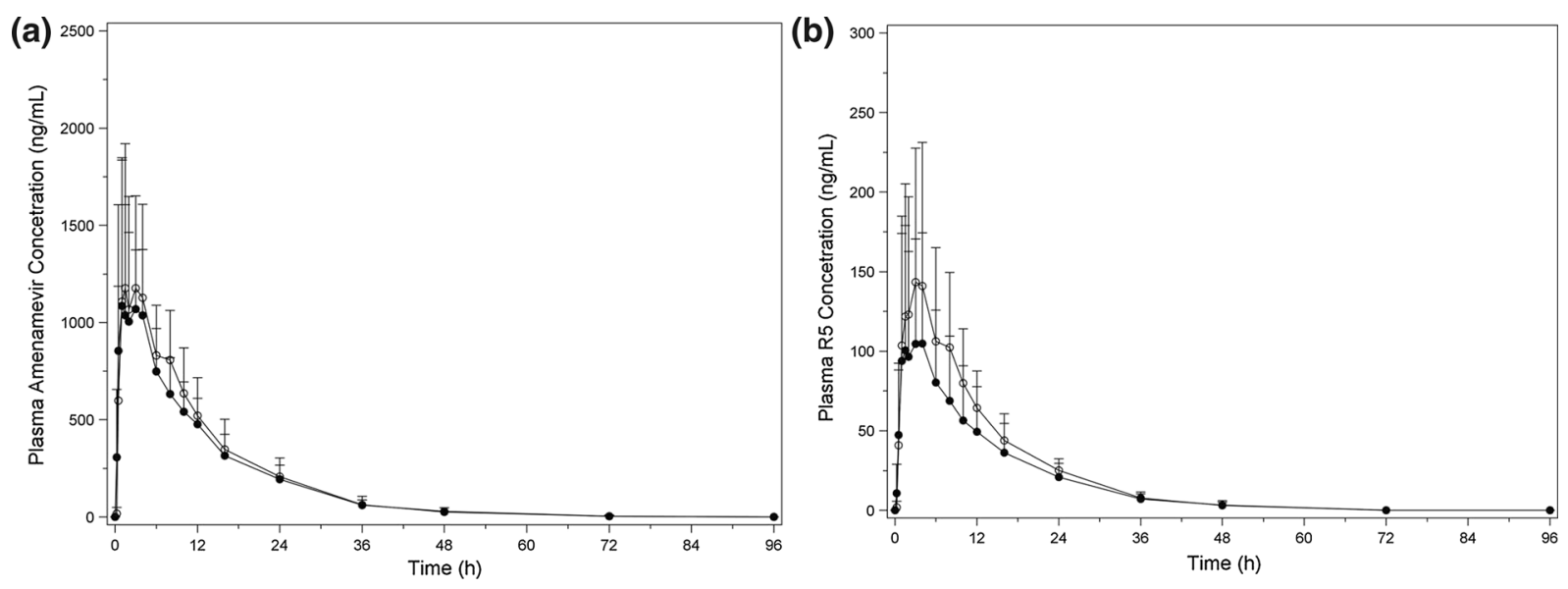

Fig. 1 Mean plasma concentration versus time curve for a amenamevir and $\mathbf{b}$ metabolite R5 in participants with normal and moderately impaired hepatic function. Open circles healthy volunteers, closed circles hepatic impairment patients

various degrees of renal impairment and normal renal function are presented in Table 3.

The mean $\mathrm{AUC}_{\mathrm{inf}}$ of amenamevir was increased by $19.8 \%, 34.7 \%$, and $78.1 \%$ in participants with mild, moderate, and severe renal impairment, respectively, compared with participants with normal renal function. Impaired renal function had a differing effect on $C_{\max }$ of amenamevir, with mean decreases of $8.1 \%$ and $1.7 \%$ in participants with mild and moderate renal impairment, respectively, and a mean increase of $17.3 \%$ in participants with severe renal impairment compared with participants with normal renal function.

The $t_{\max }$ was somewhat greater in participants with mild $(3.0 \mathrm{~h})$, moderate $(2.5 \mathrm{~h})$, and severe $(1.8 \mathrm{~h})$ renal impairment compared with that in participants with normal renal function $(1.5 \mathrm{~h})$. The $t_{1 / 2}$ of amenamevir was slightly greater in participants with mild $(8.4 \mathrm{~h})$, moderate $(9.5 \mathrm{~h})$ and severe $(9.8 \mathrm{~h})$ renal impairment compared with that in participants with normal renal function $(8.1 \mathrm{~h})$. The mean oral clearance gradually decreased in participants with mild $(21.3 \mathrm{~L} / \mathrm{h})$, moderate $(21.1 \mathrm{~L} / \mathrm{h})$, and severe $(14.9 \mathrm{~L} / \mathrm{h})$ renal impairment compared with that in participants with normal renal function $(26.1 \mathrm{~L} / \mathrm{h})$. The mean fraction of unbound amenamevir was $21.7-23.0 \%$ for all participants regardless of renal function.

Renal impairment had a greater effect on disposition of metabolite R5 than on disposition of amenamevir. The mean $\mathrm{AUC}_{\text {inf }}$ of $\mathrm{R} 5$ was increased by $47.2 \%, 86.7 \%$, and $155.5 \%$ in participants with mild, moderate, and severe renal impairment, respectively, compared with participants with normal renal function. The mean $C_{\max }$ of R5 was increased by $8.5 \%, 22.2 \%$, and $37.9 \%$ in participants with mildly, moderately, and severely impaired renal function, respectively, compared with participants with normal renal function.

There was a positive relationship between creatinine clearance and the pharmacokinetic parameters oral clearance and renal clearance for both amenamevir and R5 (Fig. 3).

The mean $A_{\text {last }}$ of amenamevir and R5 of participants with normal renal function and various degrees of impaired renal function is presented in Fig. 4. The amounts of amenamevir and R5 excreted in urine decreased with increasing renal impairment.

The percentage $\mathrm{Ae}_{\text {last }}$ of amenamevir decreased with increasing renal impairment. The mean percentage $\mathrm{Ae}_{\text {last }}$ of amenamevir decreased to $8.5 \%, 6.4 \%$, and $6.1 \%$ in participants with mildly, moderately, and severely impaired renal function, respectively, compared with $11.9 \%$ in participants with normal renal function. For R5, the mean $\mathrm{Ae}_{\text {last }}$ decreased to $6.8 \%, 3.9 \%$ and $3.0 \%$ in participants with mildly, moderately, and severely impaired renal function, respectively, compared with $7.9 \%$ in participants with normal renal function. 


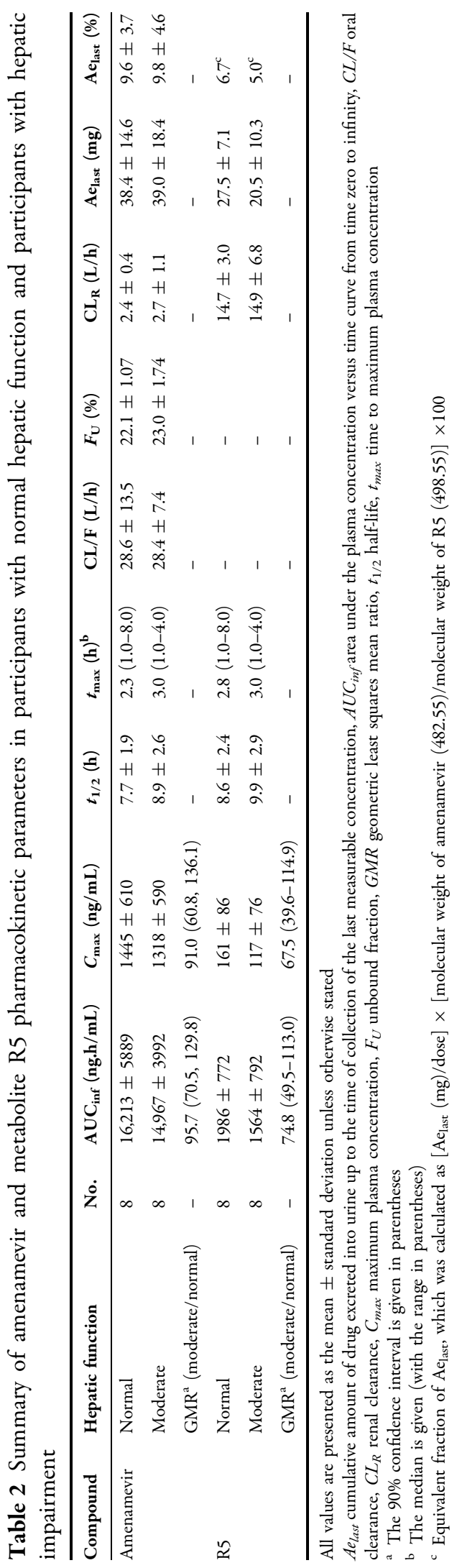




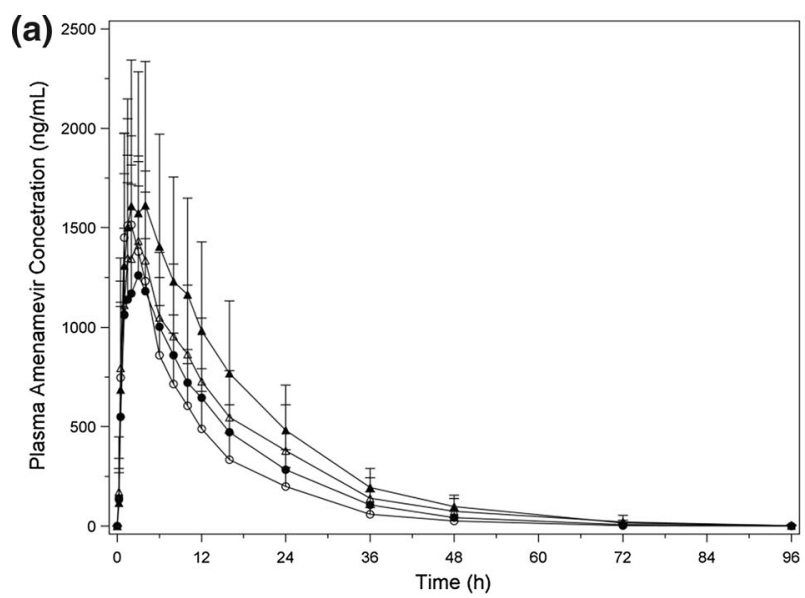

Fig. 2 Mean plasma concentration versus time curve for a amenamevir and $\mathbf{b}$ metabolite R5 in participants with normal and impaired renal function. Open circles healthy

\section{Safety}

There were no deaths or serious TEAEs, and no participants discontinued their participation because of a TEAE in either study.

In the hepatic impairment study, one of the eight participants with normal hepatic function experienced one mild adverse event (nausea). This TEAE was considered to be drug related. No participants with moderate hepatic impairment experienced a TEAE during the study. There were no clinically significant changes from the baseline for clinical laboratory tests or vital signs, and no clinically significant changes were observed for ECGs or physical examinations.

In the renal impairment study, five participants reported TEAEs. No TEAEs were reported in participants with normal renal function. One participant in the mild renal impairment group and two participants in each of the moderate and severe renal impairment groups reported TEAEs. Only one moderate adverse event (diarrhea) was reported in the severe renal impairment group, and all other events were reported as mild. One TEAE of constipation in a participant with mild renal impairment was considered to be drug related. There were no clinically significant changes from the baseline for clinical laboratory tests or vital signs, and no

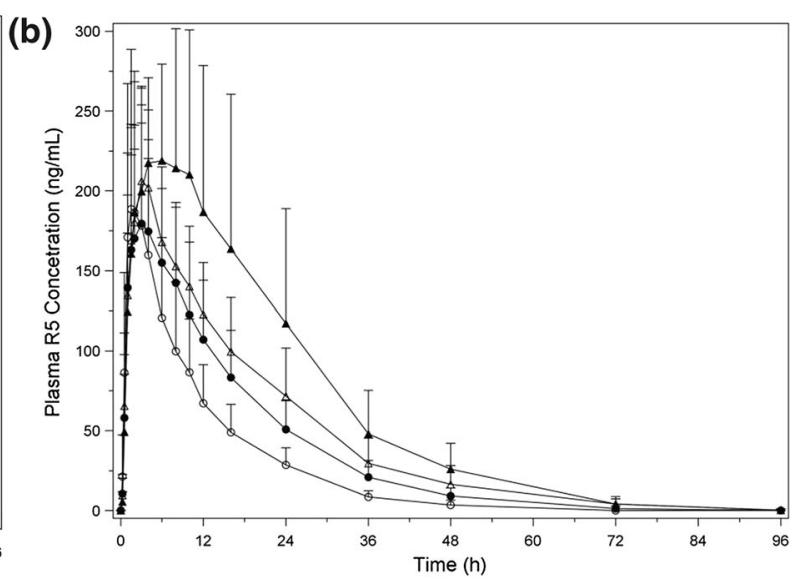

volunteers, closed circles mild renal impairment, open triangles moderate renal impairment, closed triangles severe renal impairment

clinically significant changes were observed for ECGs or physical examinations.

\section{DISCUSSION}

The viral helicase-primase inhibitor amenamevir is a nonnucleoside anti-human herpesvirus drug that was approved in Japan for treatment of herpes zoster (shingles) in 2017. Two phase 1 studies were conducted to evaluate the effects of hepatic and renal impairment on the pharmacokinetics of amenamevir.

In the hepatic impairment study, the pharmacokinetic profile of amenamevir in participants with moderate hepatic impairment was generally similar to that of participants with normal hepatic function. Amenamevir $\mathrm{AUC}_{\mathrm{inf}}$ and $C_{\max }$ geometric least squares mean ratios (i.e., moderately impaired hepatic function/ normal hepatic function expressed as percentages) were $95.7 \%(90 \%$ CI $70.5-129.8 \%)$ and 91.0\% (90\% CI 60.8-136.1\%), respectively. Although the $90 \%$ CIs for the ratios of $\mathrm{AUC}_{\text {inf }}$ and $C_{\max }$ fell outside the range of $80-125 \%$, this alteration was not considered to be clinically significant given the small number of participants. Furthermore, there was no clear relationship between hepatic function abnormalities, albumin and total bilirubin 


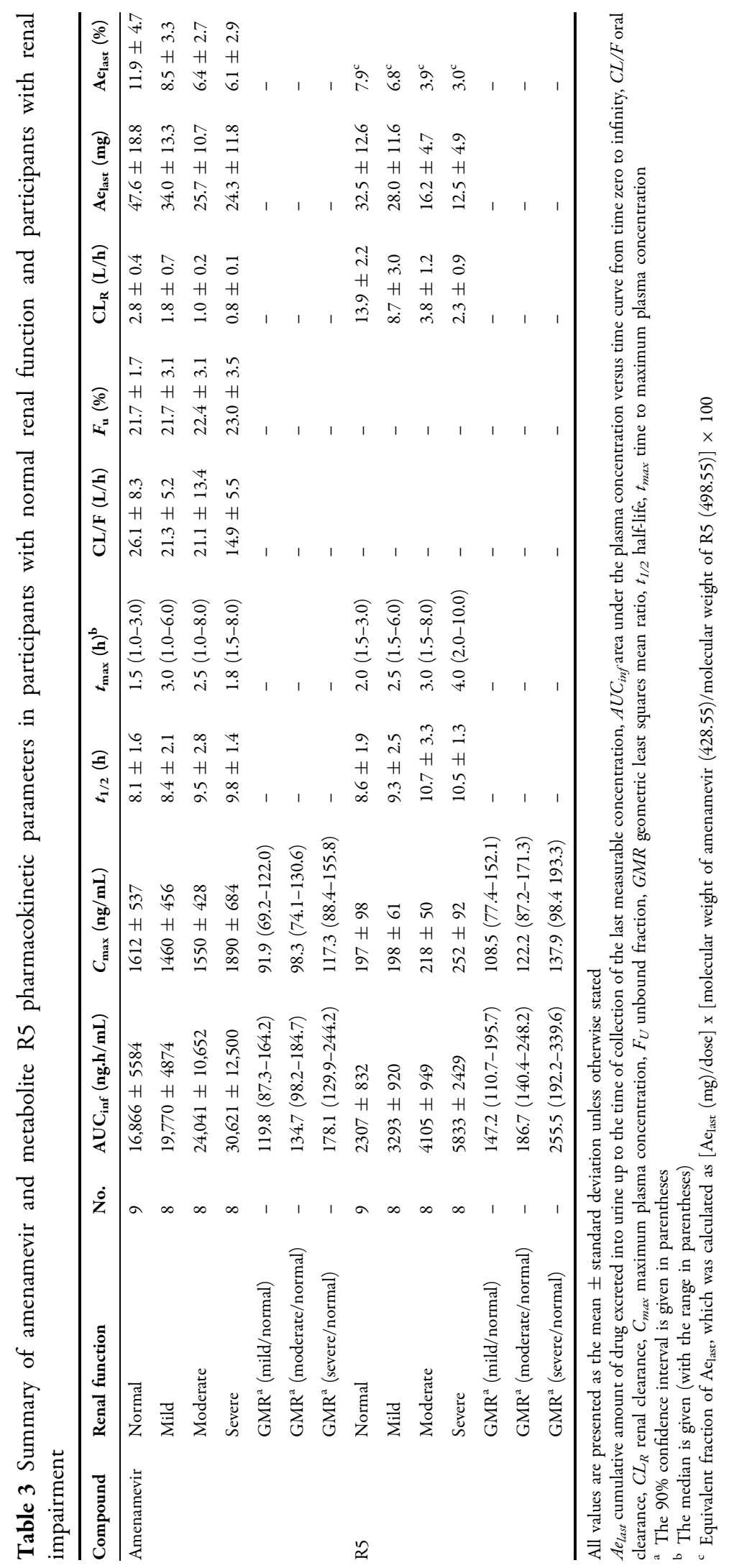



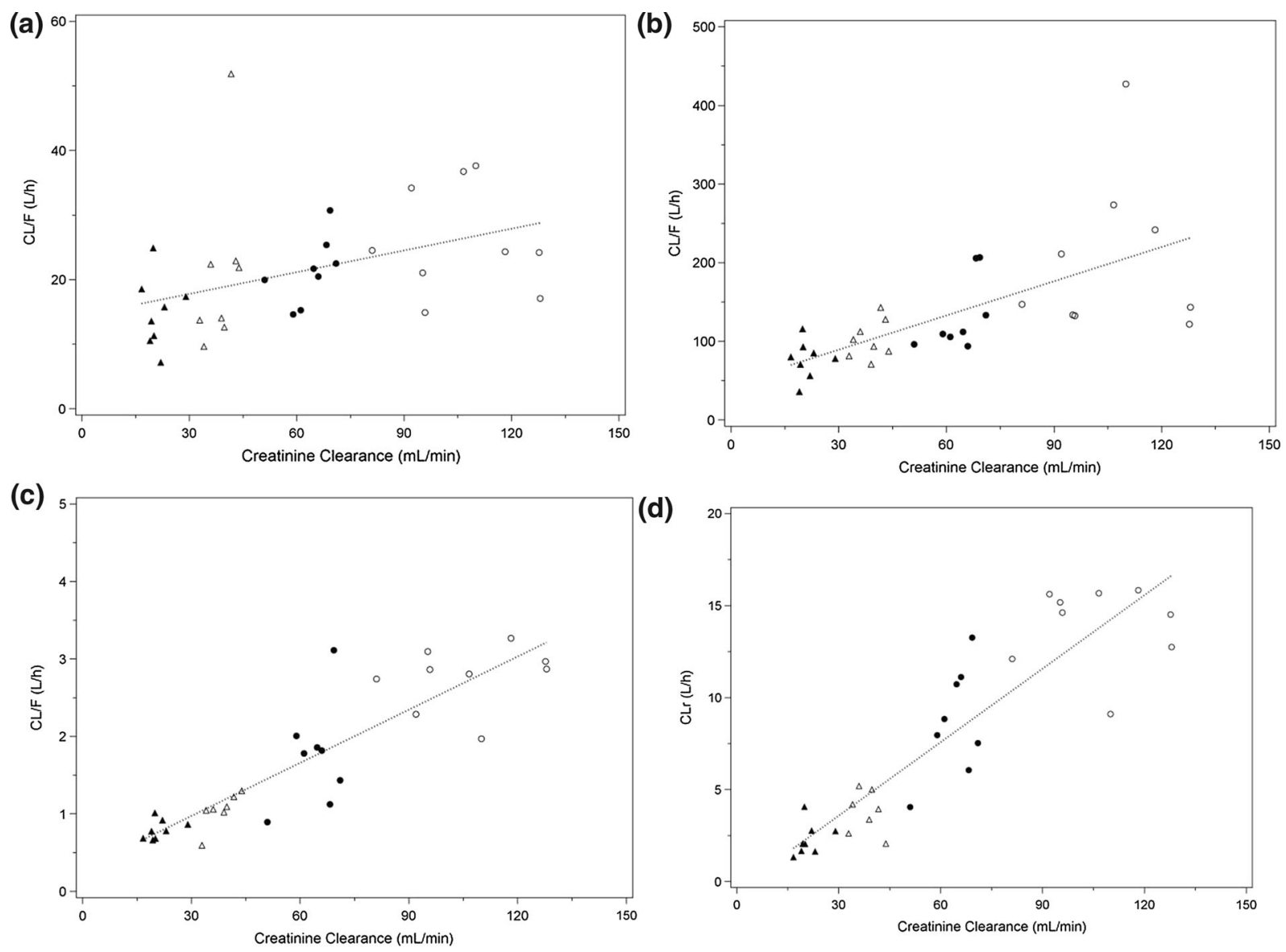

(d)

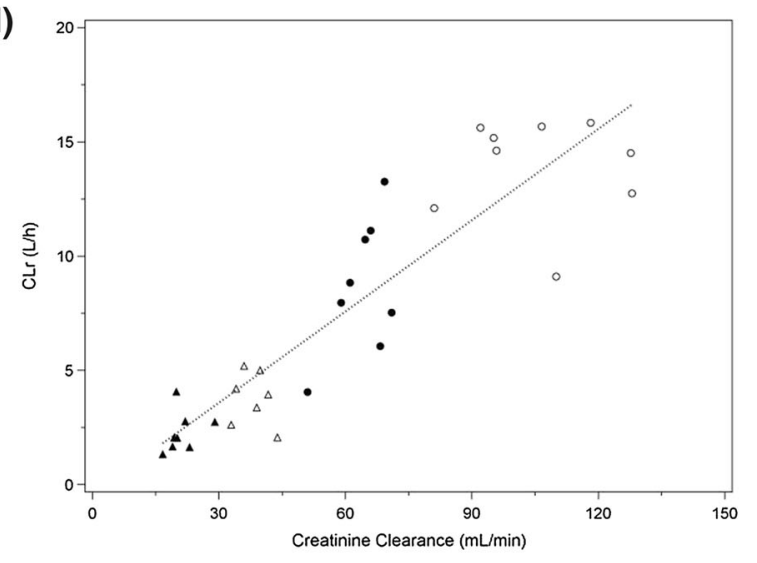

Fig. 3 Relationship between creatinine clearance and oral clearance $(\mathrm{CL} / \mathrm{F})$ for $\mathbf{a}$ amenamevir and $\mathbf{b}$ metabolite R5, and creatinine clearance and renal clearance (CLr) for c amenamevir and d R5 of participants with normal and

impaired renal function. Open circles healthy volunteers, closed circles mild renal impairment, open triangles moderate renal impairment, closed triangles severe renal impairment, dotted line regression line

levels, and the pharmacokinetic parameters $\mathrm{AUC}_{\text {inf }}$ and $C_{\max }$ for amenamevir (data not shown).

Amenamevir metabolic activity has been significantly correlated with marker enzyme activities specific for cytochrome P450 (CYP) isozymes CYP2B6, CYP2C19, and CYP3A4/5 [18], suggesting that several CYP isozymes are involved in the metabolism of amenamevir. In addition, amenamevir is partly excreted via urine. However, in the hepatic impairment study, there was no difference in the cumulative amount excreted and renal clearance of amenamevir in urine between participants with normal hepatic function and participants with moderately impaired hepatic function. This suggests that although the activity of metabolic

enzymes may be reduced in individuals with hepatic impairment, the pharmacokinetic profile of amenamevir in individuals with moderate hepatic impairment was not significantly affected.

Metabolite R5 is the major metabolite found in human plasma, and hepatic impairment had a greater effect on excretion of R5 than of amenamevir. Although it is difficult to clearly conclude reasons because of the high variability, CYP3A4/5 is the main CYP isozyme of the metabolite R5 production, and reduction of CYP3A4/5 activity for patients with hepatic impairment might be involved in the reduction of metabolite R5 production. Because R5 is not an active metabolite, the decreased exposure of 


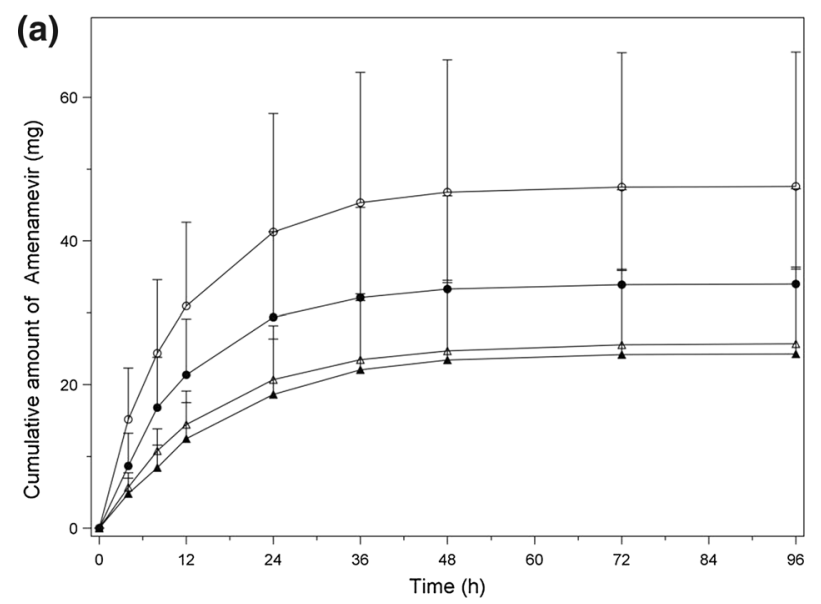

Fig. 4 Mean cumulative amount of a amenamevir and b R5 excreted in urine of participants with normal and impaired renal function. Open circles healthy volunteers,

R5 in individuals with hepatic impairment might be less clinically meaningful.

Amenamevir was safe and generally well tolerated after a single 400-mg dose. No TEAE was observed in participants with moderately impaired hepatic function. There were no clinically significant changes from the baseline for clinical laboratory tests, including liver function tests.

In the renal impairment study, increasing renal dysfunction was associated with an increase in systemic exposure to amenamevir and a reduction in renal clearance. The mean oral clearance of amenamevir in participants with mildly, moderately, and severely impaired renal function was approximately $18.4 \%$, $19.2 \%$, and $42.9 \%$ lower than that in participants with normal renal function. Renal impairment had a significant effect on $\mathrm{AUC}_{\text {inf }}$ of amenamevir, and $\mathrm{AUC}_{\text {inf }}$ was increased in participants with renal dysfunction. Impaired renal function had differing effects on $C_{\max }$ of amenamevir.

Renal impairment had a greater effect on excretion of metabolite R5 than on excretion of amenamevir. $\mathrm{AUC}_{\mathrm{inf}}$ and $C_{\max }$ of $\mathrm{R} 5$ was increased in participants with renal dysfunction. This suggests that renal elimination might be the predominant pathway for metabolite R5. Because R5 is not an active metabolite, the increased exposure of R5 in participants with

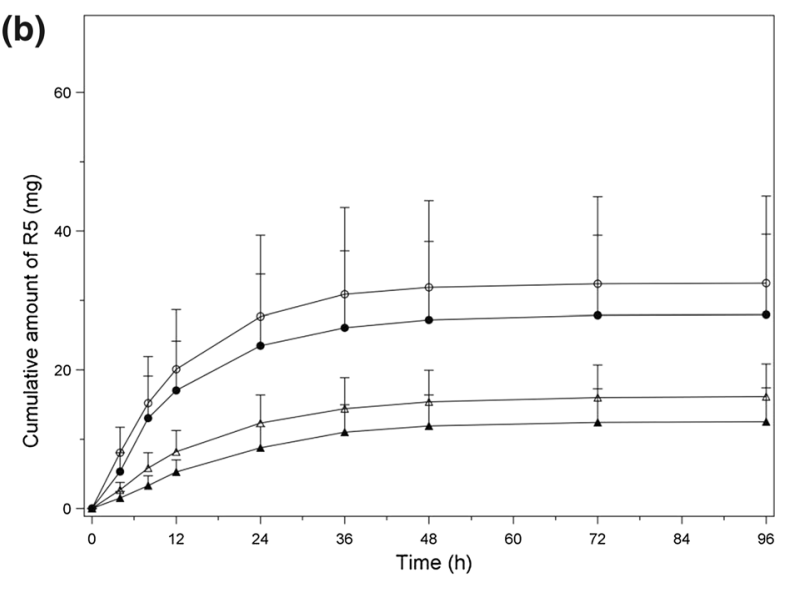

closed circles mild renal impairment, open triangles moderate renal impairment, closed triangles severe renal impairment

renal impairment might be less clinically meaningful.

In the renal impairment study, amenamevir was safe and generally well tolerated after a single 400-mg dose. There were no deaths or serious TEAEs, and no participants discontinued their participation because of a TEAE. Only one mild TEAE (constipation) in a participant with mild renal impairment was considered to be drug related. There were no clinically significant changes from the baseline for clinical laboratory tests, including renal function tests.

The recommended dosage of amenamevir for VZV is $400 \mathrm{mg} /$ day by oral administration for 7 days. Previous pharmacokinetic and safety studies of amenamevir in healthy individuals have shown that amenamevir is safe and well tolerated after a single dose (5-2400 $\mathrm{mg}$, fasted condition) and repeated doses for 7 days (300 or $600 \mathrm{mg}$, fed condition) [19]. Even though systemic amenamevir exposure was increased by $78.1 \%$ by severe renal impairment in the renal impairment study, the amount of amenamevir exposure was covered by those previous pharmacokinetic studies. Thus, it seems that repeated dosing of $400 \mathrm{mg}$ amenamevir for 7 days in patients with severe renal impairment is likely to produce no significant problems with safety.

These studies have some limitations that may affect the interpretation of the findings. As 
with most phase 1 studies, the study populations were relatively small, and randomized controlled studies with larger patient populations are needed in clinical practice.

\section{CONCLUSION}

Overall, the findings from the hepatic impairment study indicate that no dosing adjustment is required in patients with moderate hepatic impairment. Also, the findings from the renal impairment study suggest that amenamevir does not require dosage reduction in accordance with the creatinine clearance and is likely a useful option for patients with renal impairment.

\section{ACKNOWLEDGEMENTS}

Sponsorship for the studies, article processing charges, and the open access fee were funded by Astellas Pharma. All authors had full access to all of the data in these studies and take complete responsibility for the integrity of the data and accuracy of the data analysis. We thank Simone Boniface of Springer Healthcare Communications, who edited and styled the manuscript for submission. This medical writing assistance was funded by Astellas Pharma. All named authors meet the International Committee of Medical Journal Editors (ICMJE) criteria for authorship for this manuscript, take responsibility for the integrity of the work as a whole, and have given final approval to the version to be published.

Disclosures. Tomohiro Kusawake is an employee of Astellas Pharma. Donna Kowalski is an employee of Astellas Pharma. Akitsugu Takada is an employee of Astellas Pharma. Kota Kato is an employee of Astellas Pharma. Masataka Katashima is an employee of Astellas Pharma. James J. Keirns was an employee of Astellas at the time of the studies. James J. Keirns is now an independent consultant in clinical pharmacology. Michaelene Lewand was an employee of Astellas at the time of the studies. Michaelene Lewand is now an employee of AbbVie. Kenneth C. Lasseter is an employee of
Clinical Pharmacology of Miami, LLC., who were contracted by Astellas Pharma Global Development, Inc. to perform work related to the study. Thomas C. Marbury is an employee and equity owner of Orlando Clinical Research Center, who were contracted by Astellas Pharma Global Development, Inc. to perform work related to the study. Richard A. Preston is an employee of University of Miami, who were contracted by Astellas Pharma Global Development, Inc. to perform work related to the study.

Compliance with Ethics Guidelines. Before initiation of the studies, the clinical study protocols were approved by the Independent Investigational Review Board (Plantation, FL, USA) for Orlando Clinical Research Center and Clinical Pharmacology of Miami, the University of Miami Human Subjects Research Office (Miami, FL, USA) for the University of Miami, and RCRC IRB (Austin, TX, USA) for DGD Research. The studies were conducted in accordance with the ethical standards of the International Council for Harmonisation of Technical Requirements for Pharmaceuticals for Human Use "Guideline for Good Clinical Practice." All procedures followed were in accordance with the ethical standards of the responsible committee on human experimentation (institutional and national) and with the Helsinki Declaration of 1964 , as revised in 2000 . Written informed consent was obtained from all participants for their being included in these studies.

Data Availability. The data sets generated during and/or analyzed during the current study are not publicly available because these studies are small single-center studies and anonymization of these data is difficult to achieve.

Open Access. This article is distributed under the terms of the Creative Commons Attribution-NonCommercial 4.0 International License (http://creativecommons.org/licenses/ by-nc/4.0/), which permits any noncommercial use, distribution, and reproduction in any medium, provided you give appropriate credit to the original author(s) and the source, provide a link to the Creative Commons license, and indicate if changes were made. 


\section{REFERENCES}

1. Cohen JI. Herpes zoster. N Engl J Med. 2013;369(18):1766-7.

2. Johnson RW, Alvarez-Pasquin MJ, Bijl M, et al. Herpes zoster epidemiology, management, and disease and economic burden in Europe: a multidisciplinary perspective. Ther Adv Vaccines. 2015;3(4):109-20.

3. Schmader K. Herpes zoster in older adults. Clin Infect Dis. 2001;32(10):1481-6.

4. GlaxoSmithKline. ZOVIRAX ${ }^{\circledR}$ (acyclovir) capsules, tablets, suspension: prescribing information. 2005. http://www.accessdata.fda.gov/drugsatfda_docs/ label/2005/018828s030,020089s019,019909s020 lbl.pdf. Accessed 2 Aug 2017.

5. GlaxoSmithKline. VALTREX ${ }^{\circledR} \quad$ (valacyclovir hydrochloride) caplets: prescribing information. 2008. http://www.accessdata.fda.gov/drugsatfda docs/label/2008/020487s014lbl.pdf. Accessed $\overline{2}$ Aug 2017.

6. Novartis. Famvir ${ }^{\circledR}$ (famciclovir) tablets: prescribing information. 2006. http://www.accessdata.fda.gov/dru gsatfda_docs/label/2006/020363s026lbl.pdf. Accessed 2 Aug 2017.

7. Boike SC, Pue MA, Freed MI, et al. Pharmacokinetics of famciclovir in subjects with varying degrees of renal impairment. Clin Pharmacol Ther. 1994;55(4):418-26.

8. Cockcroft DW, Gault MH. Prediction of creatinine clearance from serum creatinine. Nephron. 1976;16(1):31-41.

9. Chono K, Katsumata K, Kontani T, et al. ASP2151, a novel helicase-primase inhibitor, possesses antiviral activity against varicella-zoster virus and herpes simplex virus types 1 and 2 . J Antimicrob Chemother. 2010;65(8):1733-41.

10. Crute JJ, Grygon CA, Hargrave KD, et al. Herpes simplex virus helicase-primase inhibitors are active in animal models of human disease. Nat Med. 2002;8(4):386-91.
11. Crute JJ, Mocarski ES, Lehman IR. A DNA helicase induced by herpes simplex virus type 1 . Nucleic Acids Res. 1988;16(14A):6585-96.

12. Crute JJ, Tsurumi T, Zhu LA, et al. Herpes simplex virus 1 helicase-primase: a complex of three herpes-encoded gene products. Proc Natl Acad Sci U S A. $1989 ; 86(7): 2186-9$.

13. Dodson MS, Crute JJ, Bruckner RC, Lehman IR. Overexpression and assembly of the herpes simplex virus type 1 helicase-primase in insect cells. J Biol Chem. 1989;264(35):20835-8.

14. Kleymann G, Fischer R, Betz UA, et al. New helicase-primase inhibitors as drug candidates for the treatment of herpes simplex disease. Nat Med. 2002;8(4):392-8.

15. Ohtsu Y, van Trigt R, Takama K, et al. Quantification of ASP2151 in human plasma and urine: a pitfall associated with supersaturation of analyte in urine. Chromatographia. 2017;80(2):217-27.

16. Pugh RN, Murray-Lyon IM, Dawson JL, Pietroni MC, Williams R. Transection of the oesophagus for bleeding oesophageal varices. $\mathrm{Br}$ J Surg. 1973;60(8):646-9.

17. US Food and Drug Administration. Guidance for industry: pharmacokinetics in patients with impaired renal function-study design, data analysis, and impact on dosing and labeling. 1998. http://www.fda.gov/downloads/Drugs/Guidance ComplianceRegulatoryInformation/Guidances/ UCM072127.pdf. Accessed 10 Aug 2017.

18. Katsumata K, Chono K, Kato K, et al. Pharmacokinetics and pharmacodynamics of ASP2151, a helicase-primase inhibitor, in a murine model of herpes simplex virus infection. Antimicrob Agents Chemother. 2013;57(3):1339-46.

19. Kusawake T, Keirns J, Kowalski D, et al. Pharmacokinetics and safety of amenamevir in healthy subjects: analysis of four randomized phase 1 studies. Adv Ther. 